\title{
Une approche conceptuelle
}

\section{Antoine Bailly}

\section{(2) OpenEdition}

\section{Journals}

Édition électronique

URL : http://journals.openedition.org/trema/2295

DOI : 10.4000/trema.2295

ISSN : 2107-0997

\section{Éditeur}

Faculté d'Éducation de l'université de Montpellier

\section{Édition imprimée}

Date de publication : 1 mai 1994

Pagination : 91-96

ISSN : 1167-315X

\section{Référence électronique}

Antoine Bailly, « Une approche conceptuelle », Tréma [En ligne], 5 | 1994, mis en ligne le 01 mai 1994, consulté le 01 mai 2019. URL : http://journals.openedition.org/trema/2295 ; DOI : 10.4000/ trema.2295

Ce document a été généré automatiquement le 1 mai 2019.

Trema 


\title{
Une approche conceptuelle
}

\author{
Antoine Bailly
}

1 «Arrivant à un carrefour à deux voies, un cycliste venant de l'est veut se diriger vers Montpellier. La route à gauche va vers une autre ville. La route en face aboutit à un étang. Quelle est la direction de Montpellier?». Voici un problème simple, posé en psychologie cognitive, pour traiter d'orientation dans l'espace, et qui oblige à mener une série d'opérations mentales nécessaires à sa résolution; on peut les résumer en cinq séquences :

- temps de lecture ou d'écoute de la question

- mémorisation-compréhension du problème

- mobilisation de concepts pour la construction d'une image mentale : droite, gauche, etc.

- organisation spatiale à partir de ces concepts et création d'une image mentale

- solution au problème.

2 Ce processus prendra plus ou moins de temps selon que les personnes sont « imageantes » et accoutumées à la mobilisation de concepts spatiaux ou qu'elles ne le sont pas. Cet exemple classique montre toute l'utilité de la démarche conceptuelle dans l'enseignement de la géographie.

\section{Les concepts, systèmes mentaux de références et tremplins pédagogiques}

3 Comme toute forme de connaissance, nos connaissances spatiales sont acquises grâce à des "catégories mentales", véritables cadres de formalisation et de codage, structurés lors de notre développement. Ce processus évolue par assimilation -intégration des données de l'expérience clans nos catégories mentales- et par accommodation transformation des catégories en fonction de données nouvelles.

4 La connaissance régionale repose donc sur des représentations à partir de cadres conceptuels, évolutifs selon nos apprentissages. Ces concepts, idées mentales abstraites, permettent de passer de la simple description à la représentation régionale et de la 
rendre intelligible. Ne cherchons pas à chasser concepts et représentations..., ils reviennent au galop! Gardons en mémoire que lorsqu'on parle de représentation, il s'agit toujours de la représentation de quelque chose (une région), mais aussi pour quelqu'un (habitant, touriste...), comme l'ont bien montré Durkheim et Moscovici (1961). De ce fait la démarche géographique se doit de révéler l'espace vécu (espace égocentré) au niveau des expériences directes, et les espaces représentés (espaces exocentrés) à travers des connaissances indirectes.

Les rapports des élèves à l'espace se déroulent à cinq niveaux de représentation géographique, différenciés par leurs échelles. Selon R. Hérin (1984), au niveau proche, il s'agit de «l'échelle locale des perceptions de l'espace quotidien de résidence et de travail », au-delà on parle de "l'échelle de la petite région que délimitent les pratiques commerciales et familiales ", de « l'échelle régionale formée par l'histoire et aujourd'hui associée au rayonnement d'une ville ", de "l'échelle nationale des décisions politiques " et de "l'échelle internationale des flux de population, de capitaux». L'effet «zoom» permet le passage d'une échelle à une autre; il est en effet indispensable, en géographie, de ne pas raisonner à une seule échelle, chacune relevant de logiques spatiales différentes.

Le concept de région se situe aux trois échelles intermédiaires, au-delà des métastructures élémentaires du local; ce qui signifie que la région constitue une représentation mentale, plus imagée et idéale que vécue, même si ces représentations se hiérarchisent solidement à partir de lieux de référence (axes structurants, nœuds, limites...) et en termes d'intériorité et d'extériorité (espace égocentré, espace exocentré). De ce fait, aborder l'enseignement de la géographie régionale suppose une réflexion fondamentale sur les échelles et concepts à mobiliser. Nous en proposons une liste dans le tableau 1, en sachant que ces concepts sont évolutifs et que cette liste n'est ni exhaustive, ni définitive, en sachant également qu'ils nous aident à construire un savoir qui répond à six grandes questions: où, combien, jusqu'où, qui, comment, pourquoi? Le champ conceptuel mobilisé varie ainsi suivant les échelles spatiales et temporelles utilisées et les grandes questions que se posent les géographes; il illustre notre capacité à réfléchir en termes théoriques sur des thèmes spatiaux, présentés dans de nombreux pays par des programmes (contenus) insistant sur les territoires et leur organisation spatiale. 
Tableau I - Questions et concepts géographiques

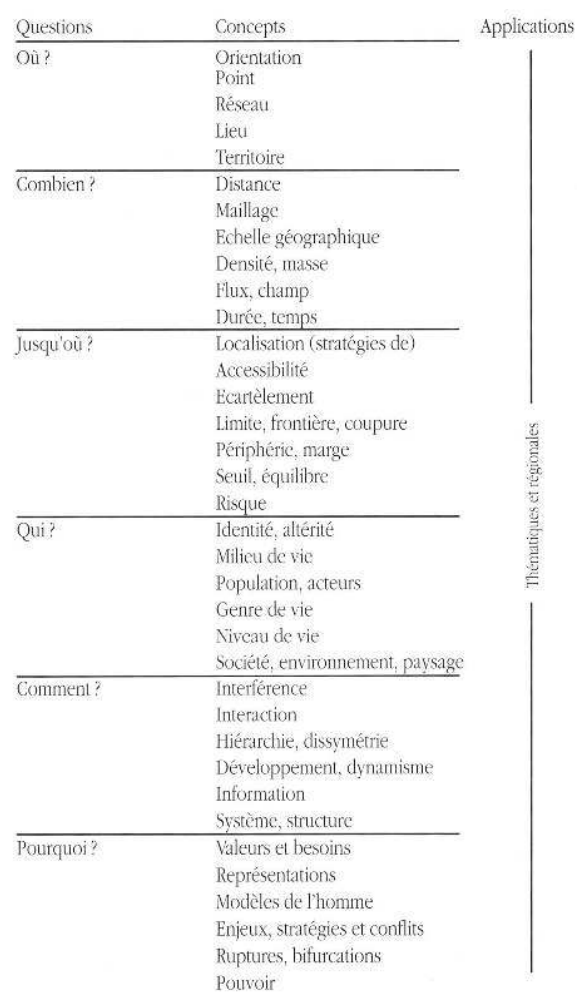

Source : R. Ferras, H. Beguin et A. Bailly

\section{Une progression en spirale}

7 Afin d'utiliser ces concepts comme supports pédagogiques, il est indispensable de dégager les représentations que l'élève a déjà de la réalité spatiale. En effet, il est acteur géographique, consommateur d'espace, sur lequel et dans lequel il mobilise ses représentations en fonction de ses objectifs; Moscovici parlait de «noyau dur de la connaissance ", organisé en schémas coordonnés, structurés, qu'illustrent bien les cartes en chorèmes. Les concepts doivent être articulés à partir de ce «noyau dur ", sous peine de confusion dans le message, car les élèves ne sont pas égaux face à l'imagerie et à la conceptualisation. M. Denis (1989) nous rappelle que les différences individuelles se manifestent dès le jeune âge : « deux études menées sur des enfants âgés de 7 à 11 ans, ont montré que les enfants imageants se souviennent mieux des textes concrets que les autres jeunes. Inversement, ces derniers se souviennent mieux des textes abstraits... » (p. 189).

8 Afin de transformer durablement les représentations de l'élève, une progression en spirale, qui permet d'aborder les concepts à des niveaux de complexité croissants, constitue la base pédagogique de l'enseignement. Ces structures élémentaires sont révélées tout d'abord de façon simple, puis approfondies. La spirale de progression dans les apprentissages part de l'hypothèse que l'élève ne passera d'une boucle à l'autre que lorsqu'il aura maîtrisé la phase précédente de la représentation conceptuelle. L'enseignement se construit progressivement autour des mêmes concepts. 
9 L'évaluation consiste à révéler les potentialités de l'élève et leur évolution; d'où des méthodes de type formatif pour suivre la progression dans la maîtrise des concepts. En effet, en matière de représentation, il n'y a pas de vrai ou de faux, mais mobilisation de propriétés fonctionnelles qui sont liées au cognitif et à l'affectif. Cette évaluation ouvre une voie de liberté, condition essentielle pour la formation de citoyens susceptibles d'être intéressés au monde des autres et d'écouter d'autres systèmes conceptuels.

\section{Donner un sens à la géographie régionale}

Les géographes et leurs élèves font partie des sociétés dont ils reflètent les idéologies et les valeurs; d'où la nécessité, en géographie régionale, d'expliciter les concepts de base de la représentation et de les faire évoluer.

11 Le rôle de la géographie régionale, en tant qu'élément de la connaissance et de l'apprentissage, consiste donc :

- à révéler les capacités spatiales et temporelles des élèves, à des niveaux de complexité croissants et à accroître leur capacité de réflexion. La géographie est une éducation des yeux, des sens et de la conscience spatiale,

- à identifier les concepts évolutifs qui permettent d'expliciter les représentations régionales, de comprendre les espaces des sociétés et de faire une évaluation prospective des territoires,

- à acquérir un savoir géographique qui peut être articulé à partir de grandes lois et de modèle spatiaux,

- à articuler le réel et l'imaginaire, le quotidien et le fantastique, dans un contexte de représentation sociale et idéologique du monde.

12 Après avoir passé des années à "gommer » toute trace de subjectivité, la géographie régionale retrouve une voie riche que nous rappellent cinq grands principes, destinés à éviter le caractère desséchant des énumérations et des modèles simplificateurs (Bailly et al., 1991, chap. 1).

Le principe existentiel : les géographes s'intéressent aux hommes en société, mais font aussi partie de sociétés dont ils reflètent les idéologies. D'où la nécessité de l'explicitation des idéologies et des concepts avec lesquels ils explorent la connaissance et la présentent à leurs élèves.

Le principe de représentation : la région en soi n'est pas l'objet d'étude, puisque le rôle objectif n'existe que grâce à nos concepts. La connaissance géographique repose sur la représentation de phénomènes; l'image mentale d'une région constitue en fait une représentation de représentation puisque le concept de région est déjà une représentation.

Le principe imaginaire : toute proposition de représentation géographique est une image, c'est-à-dire un modèle simplifié ; à la géographie régionale de reconstruire " par le bas » ces régions en tenant compte des imaginaires individuels et collectifs, pour aider l'élève à progresser dans sa construction du monde.

16 Le principe de création : la représentation d'une région consiste en la création de schémas pertinents qui nous renvoient à nos idéologies et à la manière de la structurer. La géographie " par le bas » favorise la pédagogie de création en utilisant « le noyau dur » des connaissances. 
Le principe de rétroaction : les représentations des régions se nourrissent des pratiques et inversement. Ainsi, une société qui crée son milieu de vie ne le fait pas seulement à travers ses rapports de production, mais grâce aux représentations (idéologies, valeurs) propres à cette société.

Grâce à cette approche, utilisant concepts et représentations, la géographie régionale devient l'une des branches les plus fécondes de la discipline, qui nous conduit à valoriser de nouveaux concepts, de nouvelles sources de représentations et de nouveaux outils. Tel est le pari à gagner pour redonner à cette géographie sa part de rêve et d'imaginaire tant dans la construction mentale que dans les représentations graphiques régionales. La géographie régionale redevient alors cette science vivante qui étudie les territoires de l'humanité.

\section{BIBLIOGRAPHIE}

Albertini J-M., 1992, La pédagogie n'est plus ce qu'elle sera, Paris, Seuil.

André Y. et al, 1989, Représenter l'espace ; l'imaginaire spatial à l'école, Paris, Anthropos.

André Y. et al., Modèles graphiques et représentations spatiales. Paris, Anthropos.

Bailly A., Ferras R. ; Pumain D. (directeurs), 1992, Encyclopédie de géographie, Paris, Economica.

Bruner J., 1960, The process of education, Harvard, Harvard University Press.

Brunet R., 1990, Le territoire dans les turbulences, Montpellier, RECLUS.

Brunet R., Ferras R., Théry H., 1992, Les mots de la géographie, Montpellier, RECLUS.

Clary M., Dufau G., Durand R., Ferras R., 1988, Cartes et modèles à l'école, Montpellier, RECLUS.

Denis M., 1989, Image et cognition, Paris, P.U.F.

Fremont A., Herin R., Chevalier J., Renard J., 1984, Géographie sociale, Paris, Masson.

Moscovici S., 1961, La psychanalyse, son image, son public, Paris, P.U.F.

\section{AUTEUR}

\section{ANTOINE BAILLY}

Professeur à l'université de Genève 